\title{
Purification of biohydrogen from fermentation gas mixture using two-stage chemical absorption
}

\author{
Nor Azira Abdul Muin ${ }^{1,}$, and Umi Aisah Asli ${ }^{2 *}$ \\ ${ }^{1}$ School of Chemical and Energy Engineering, Faculty of Engineering, Universiti Teknologi \\ Malaysia, 81310 Johor Bahru, Johor, Malaysia. \\ ${ }^{2}$ Centre of Hydrogen Energy, Institute of Future Energy, Universiti Teknologi Malaysia, 81310 Johor \\ Bahru, Johor, Malaysia.
}

\begin{abstract}
Research on biohydrogen production via fermentation process has shown a tremendous progress for the past few years. As biohydrogen production is being established, the purification of biohydrogen should consider the process flow for future application. This paper presents an experimental study of biohydrogen purification using two-stage chemical absorption. The research work focuses on carbon dioxide $\left(\mathrm{CO}_{2}\right)$ removal, which is a major unwanted fermentation gas product via activated methyldiethanolamine (MDEA) and caustic $(\mathrm{NaOH})$ in two-stage chemical absorption. The experiment was conducted at low pressure of $1 \mathrm{bar}$ and normal room temperature of $29^{\circ} \mathrm{C}$ using a ratio of $1: 1$ of $\mathrm{CO}_{2}: \mathrm{H}_{2}$ standard gas mixture as the feed. In the first stage, $40 \mathrm{wt} . \%$ MDEA was activated by using piperazine (PZ) with the concentration between 2 and $10 \mathrm{wt} . \%$, whereas 20 wt. $\% \mathrm{NaOH}$ was used in the second stage. It was found that 6 wt. $\%$ of $\mathrm{PZ}$ was required to fully activate $40 \mathrm{wt}$. \% MDEA, which resulted in $79 \% \mathrm{CO}_{2}$ removal. To improve $\mathrm{CO}_{2}$ removal, a gas distributor and wire mesh packed were used to create gas bubbles at higher geometrical surface. The experimental study successfully removed $99.59 \%$ of the total $\mathrm{CO}_{2}$, producing $>99 \mathrm{~mol} \%$ hydrogen gas purity from the second stage that used 20 wt. $\% \mathrm{NaOH}$.
\end{abstract}

\section{Introduction}

Hydrogen $\left(\mathrm{H}_{2}\right)$ has been identified as a potential secondary source of energy, which is also termed as an energy carrier. The use of $\mathrm{H}_{2}$ can reduce the dependency on fossil fuels. $\mathrm{H}_{2}$ has the highest energy per unit mass of any known fuel. The energy per unit mass of $\mathrm{H}_{2}$ is 141.9 $\mathrm{J} \mathrm{kg}^{-1}$, which is three times higher than gasoline that contains $47.4 \mathrm{~J} \mathrm{~kg}^{-1}$ of energy [1]. $\mathrm{H}_{2}$ produces only water, which makes it clean energy. There are many methods to produce $\mathrm{H}_{2}$. For example, steam methane reforming, coal gasification, pyrolysis, biophotolysis, and fermentation. $\mathrm{H}_{2}$ produced through fermentation has been the focus of many researchers as this process can utilise renewable resources such as biomass as the raw material, as well as it is environmentally friendly and has low energy consumption. From the literature, the 
optimum biohydrogen production can be obtained via combination of dark and photofermentation processes. Morsy [2] produced a maximum of $56 \% \mathrm{H}_{2}$ and $44 \% \mathrm{CO}_{2}$ by integrating dark and photofermentation from sorghum starch. However, for biohydrogen to be used as a fuel for vehicle, it has to meet the ISO 14687-2 Hydrogen Fuel Specification that requires the minimum $\mathrm{H}_{2}$ purity of $99.97 \mathrm{~mol} \%$ [3]. Four techniques for purifying crude $\mathrm{H}_{2}$ are cryogenic separation, absorption, adsorption, and membrane separation. These methods have been commercially used to purify conventional $\mathrm{H}_{2}$. Biohydrogen is normally produced at the temperature between 30 and $60{ }^{\circ} \mathrm{C}$ and atmospheric pressure. Thus, chemical absorption is the most suitable technique for biohydrogen purification because it can be operated at low temperature and pressure.

Amine is one of the common solvents used in chemical absorption. Chemically, amine is a compound that contains nitrogen atom attached to hydrocarbon chain and/or hydrogen atom. From the industrial perspective, Kohl [4] expressed amine as a molecule that contains amino (-NH) group with at least one hydroxyl $(-\mathrm{OH})$ group attached to it. The general functions of a hydroxyl group are to reduce the vapour pressure of a solvent so that it will not be easily vapourised and to increase the solvent solubility in water. Meanwhile, an amino group will create an alkaline environment in a solvent for the extraction of acid gases. In general, amines can be divided into primary, secondary, and tertiary amines. Primary amines are those with a nitrogen atom that is directly attached with two hydrogen atoms. For secondary amines, only one hydrogen atom is directly attached to nitrogen. In tertiary amines, no hydrogen atom is directly attached to nitrogen.

In $\mathrm{CO}_{2}$ removal, tertiary amines such as methyldiethanolamine (MDEA) is normally used due its superiority compared to primary and secondary amines in terms of $\mathrm{CO}_{2}$ loading capacity, less energy requirement for regeneration, thermal and oxidative stability (i.e., less degrade), and minimum amine loss due to low vapour pressure. The drawback of tertiary amines is slower absorption rate than primary and secondary amines. This is because it cannot react directly with $\mathrm{CO}_{2}$. It must be hydrolysed before it can react with MDEA. The addition of an activator in the form of primary or secondary amine could overcome the drawback. The most commonly used activator is activated MDEA or piperazine (PZ)MDEA mixture [5]. Hidayu [6] reported $\mathrm{CO}_{2}$ loading of PZ-MDEA mixture of 1.32 mole $\mathrm{CO}_{2}$ per mole amine, whereas the $\mathrm{CO}_{2}$ loading of MDEA alone was 0.83 mole $\mathrm{CO}_{2}$ per mole amine.

In this work, two-stage chemical absorption was adopted for the purification of biohydrogen. The biohydrogen was modelled by mixing pure $\mathrm{H}_{2}$ and $\mathrm{CO}_{2}$. The main objective was to absorb $\mathrm{CO}_{2}$ for $\mathrm{H}_{2}$ purification and achieve $99.9 \%$ purity. In the first stage, MDEA was used with the activation of $\mathrm{PZ}$ whereas in the second stage, the modelled biohydrogen gas was further purified with a caustic wash $(\mathrm{NaOH})$.

\section{Methodology}

In this experiment, $50 \mathrm{~mol}^{2} \mathrm{H}_{2}$ and $50 \mathrm{~mol}^{2} \mathrm{CO}_{2}$ gas mixture was used. The ratio of mixture was chosen based on the common highest production obtained from the literature review. As for chemical solvents, $40 \mathrm{wt}$. \% MDEA activated with different concentrations of $\mathrm{PZ}$ and 20 wt. $\% \mathrm{NaOH}$ were utilised. The experimental setup is shown in Figure 1. 


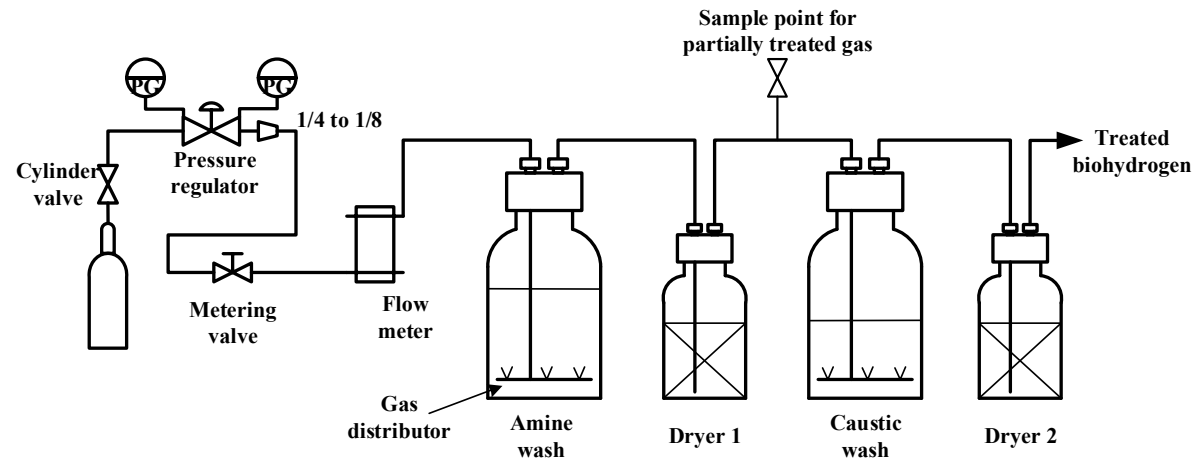

Fig. 1. Schematic diagram of experimental setup.

The experimental study was conducted in three parts as follows:

a) Part 1: In assessing the effect of PZ concentration in MDEA solution towards $\mathrm{CO}_{2}$ removal, only amine wash and dryer 1 were used. In this part, $40 \mathrm{wt}$ \% MDEA activated with different PZ concentrations $(2,4,6,8$, and $10 \mathrm{wt} . \%)$ were studied. In this part only, a gas distributor of $10.4 \mathrm{~mm}^{2}$ was used.

b) Part 2: Once the right amount of PZ required to activate 40 wt. \% MDEA had been determined in Part 1 , the effect of total surface contact area towards $\mathrm{CO}_{2}$ removal was studied by using three different gas distributors that produced geometrical gas bubble area of $10.4,7.7$, and $6.3 \mathrm{~mm}^{2}$, respectively. In this part, only amine wash and dryer 1 were used.

c) Part 3: In this final part of the experiment, the aim was to further improve $\mathrm{CO}_{2}$ removal of the latter part by utilising packing in amine wash and the use of caustic wash as the second stage. The study was conducted for gas distributors of 10.4 and $6.3 \mathrm{~mm}^{2}$ only. The packing specifications are shown in Table 1.

Table 1. Packing specifications.

\begin{tabular}{|l|c|}
\hline Packing Type & Commercial stainless steel spiral ball \\
\hline Packed Diameter $(\mathrm{m})$ & 0.095 \\
\hline Packed Height $(\mathrm{m})$ & 0.1058 \\
\hline Surface Area $\left(\mathrm{m}^{2}\right)$ & 0.3948 \\
\hline Volume $\left(\mathrm{m}^{3}\right)$ & 0.00075 \\
\hline Ratio of Surface Area to Volume $\left(\mathrm{m}^{2} \mathrm{~m}^{3}\right)$ & 526 \\
\hline
\end{tabular}

\section{Results and discussion}

Figure 2 presents the results from Part 1 of the experimental study. There are two key points to be highlighted. First, in the absence of PZ as an activator, the process did not achieve any $\mathrm{CO}_{2}$ removal. However, as $2 \mathrm{wt}$. \% PZ was used to activate $40 \mathrm{wt}$. \% MDEA, it significantly improved $\mathrm{CO}_{2}$ removal by $40 \%$. This is because $\mathrm{CO}_{2}$ does not react directly with a tertiary amine such as MDEA. On the other hand, PZ is a secondary amine that reacts with $\mathrm{CO}_{2}$ to form carbamate, which later takes part in base-catalysed hydration reaction between $\mathrm{CO}_{2}$ and MDEA, producing bicarbonate and protonated MDEA. 


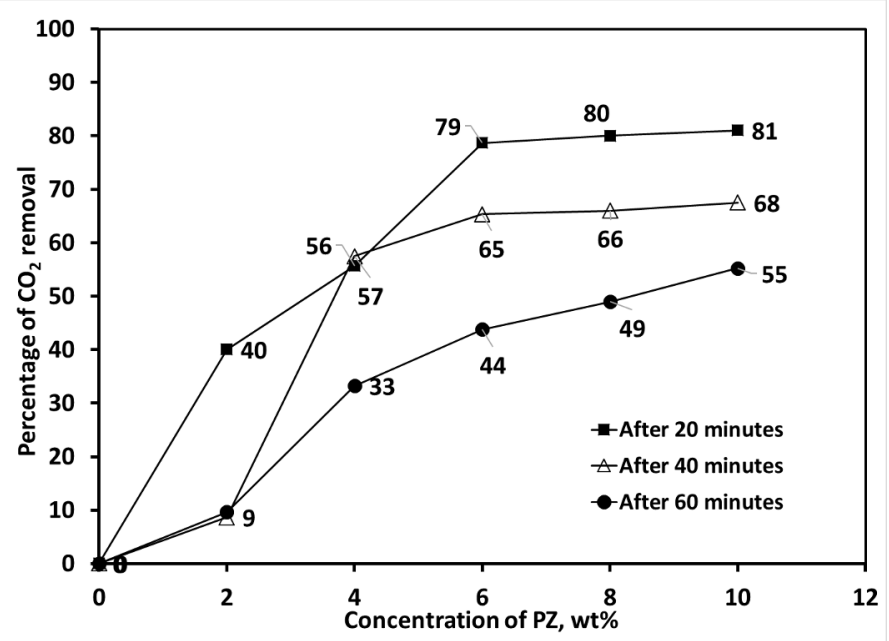

Fig. 2. The effect of 40 wt. \% MDEA activated with different PZ concentrations $(2,4,6,8$, and 10 wt. $\%)$ towards $\mathrm{CO}_{2}$ removal.

Secondly, $\mathrm{CO}_{2}$ removal improved significantly as the amount of PZ in MDEA was increased to 4 and 6 wt. \%, where the removal was $56 \%$ and $79 \%$, respectively. However, increasing $\mathrm{PZ}$ concentration beyond 6 wt. \% had a minimal effect on $\mathrm{CO}_{2}$ removal. For example, by increasing PZ concentration from 6 to $10 \mathrm{wt} \%$, only $2 \%$ improvement of $\mathrm{CO}_{2}$ removal was observed. This is because at $6 \mathrm{wt} . \% \mathrm{PZ}$, there is a sufficient amount of PZ to transport $\mathrm{CO}_{2}$ from the gas phase into the MDEA phase. As commented by Ibrahim et al. [7], once there is enough $\mathrm{PZ}$ molecules to transport $\mathrm{CO}_{2}$, any increase in $\mathrm{PZ}$ concentration will not improve $\mathrm{CO}_{2}$ removal significantly. Alvis et al. [8] in their simulation study on the effect of $\mathrm{PZ}$ concentration in MDEA stream towards $\mathrm{CO}_{2}$ removal also raised the same indication. In their study, at 4 wt. \% PZ concentration, $99.68 \%$ of $\mathrm{CO}_{2}$ removal was achieved via a multi-stage absorption process. The concentration was lower by 2 wt. \% compared to this study.

Based on Figure 3, at $20 \mathrm{~min}$ of experimental time, $\mathrm{CO}_{2}$ removal with the gas distributor of $6.3 \mathrm{~mm}^{2}$ was $67 \%$. However, as the surface area was increased to $7.7 \mathrm{~mm}^{2}$, which was an increase of $22 \%$, the percentage of $\mathrm{CO}_{2}$ removal increased to $72 \%$. Increasing the surface area by $65 \%$ from 6.3 to $10.4 \mathrm{~mm}^{2}$ improved $\mathrm{CO}_{2}$ removal from $67 \%$ to $79 \%$. This indicates that $\mathrm{CO}_{2}$ removal can be improved by increasing the surface area. 


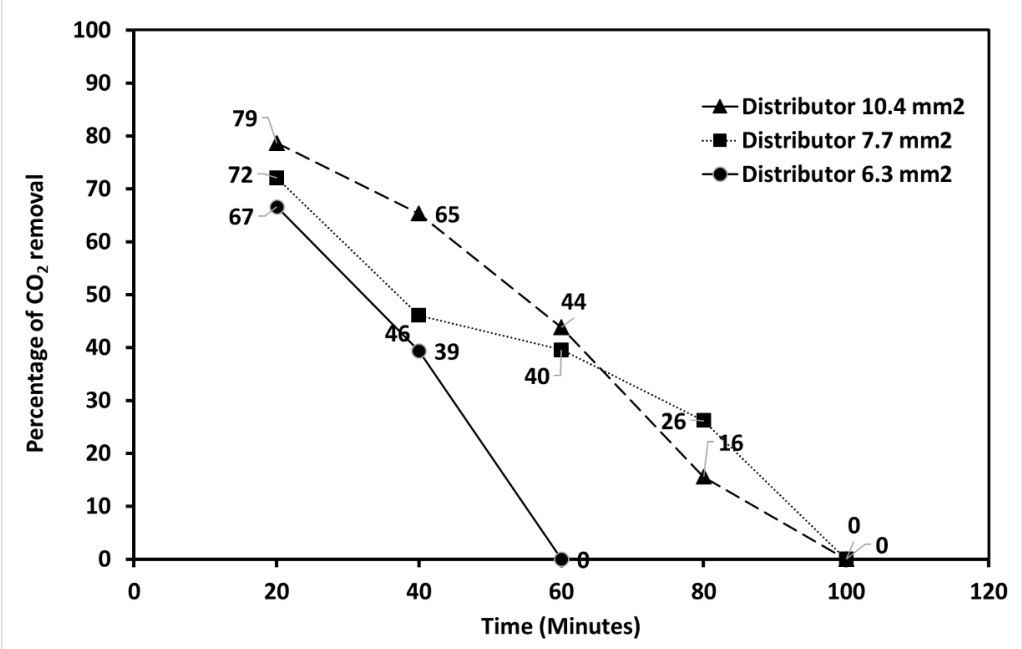

Fig. 3. Effect of total geometric surface area towards $\mathrm{CO}_{2}$ removal (amine used $=40 \mathrm{wt}$. $\%$ MDEA activated with 6 wt. \% PZ).

As the experiment was further continued, the gas distributor of $6.3 \mathrm{~mm}^{2}$ was no longer able to reduce $\mathrm{CO}_{2}$ at $60 \mathrm{~min}$ of running time. For the gas distributors of 7.7 and $10.4 \mathrm{~mm}^{2}$, $\mathrm{CO}_{2}$ removal achieved the maximum level at $100 \mathrm{~min}$ of running time. Based on the running time, it seemed a larger surface area prolonged $\mathrm{CO}_{2}$ removal. Note that the same concentration of amine mixture was used for all three gas distributors and it was not replenished during the experiment. It could be anticipated that a lower surface area gas distributor should have longer running experimental time compared to higher surface area since it absorbs less $\mathrm{CO}_{2}$. However, the opposite behaviour was observed in this experiment. The possible explanation for this behaviour relies on how gas bubbles are created by gas distributors. The gas distributor with surface area of $10.4 \mathrm{~mm}^{2}$ consists of four holes with the diameter of $0.91 \mathrm{~mm}$ each, whereas the gas distributor with surface area of $7.7 \mathrm{~mm}^{2}$ is made of five holes with $0.70 \mathrm{~mm}$ diameter each, and the gas distributor with surface area of $6.3 \mathrm{~mm}^{2}$ has eight holes with $0.50 \mathrm{~mm}$ diameter each. In short, a gas distributor with a smaller surface area creates smaller gas bubbles than a gas distributor with a larger surface area. Due to this, it is suspected that smaller bubbles have higher velocity than bigger bubbles. This will reduce the residence time of the gas phase, hence reducing $\mathrm{CO}_{2}$ removal. A detailed study might be required to prove this claim.

Figure 4 shows $\mathrm{CO}_{2}$ removal with two different sizes of distributor at the amine wash stage with packing as carried out in Part 3 of the experiment. There are two key points to be highlighted. First, the utilisation of packing improved $\mathrm{CO}_{2}$ removal as compared to Figure 3 , which only utilised a gas distributor. For the gas distributors with surface area of 10.4 and $6.3 \mathrm{~mm}^{2}$, the packing improved $\mathrm{CO}_{2}$ removal by $6 \%$ and $14 \%$, respectively. Second, as shown in Figure 4, despite different gases were used, the trend of $\mathrm{CO}_{2}$ removal was almost similar, unlike Figure 3 that clearly shows different performance for different gas distributors. This indicates that the utilisation of packing has more significant effect towards $\mathrm{CO}_{2}$ removal as compared to manipulating geometrical surface area. 


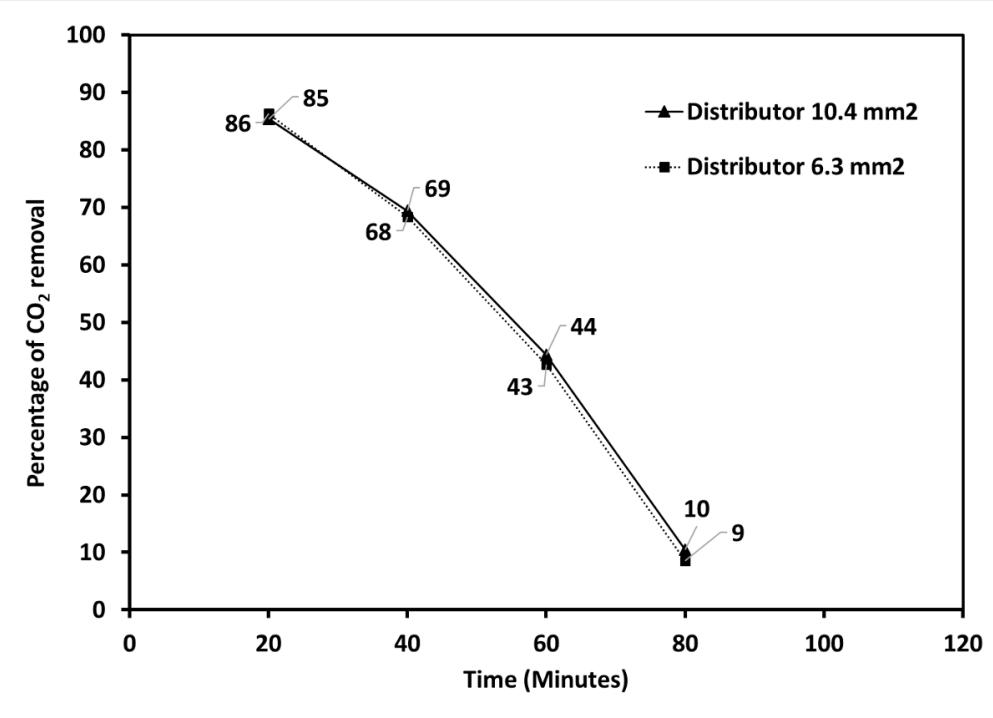

Fig. 4. $\mathrm{CO}_{2}$ removal by utilising packing at amine wash.

Figure 5 presents $\mathrm{CO}_{2}$ removal obtained from the continuation of experimental study, with 6 wt. \% PZ in 40 wt. \% MDEA was used in the first stage and 20 wt. \% NaOH was used in the second stage. The addition of $\mathrm{NaOH}$ in the second stage significantly improved $\mathrm{CO}_{2}$ removal and also prolonged high $\mathrm{CO}_{2}$ removal as compared to the amine stage.

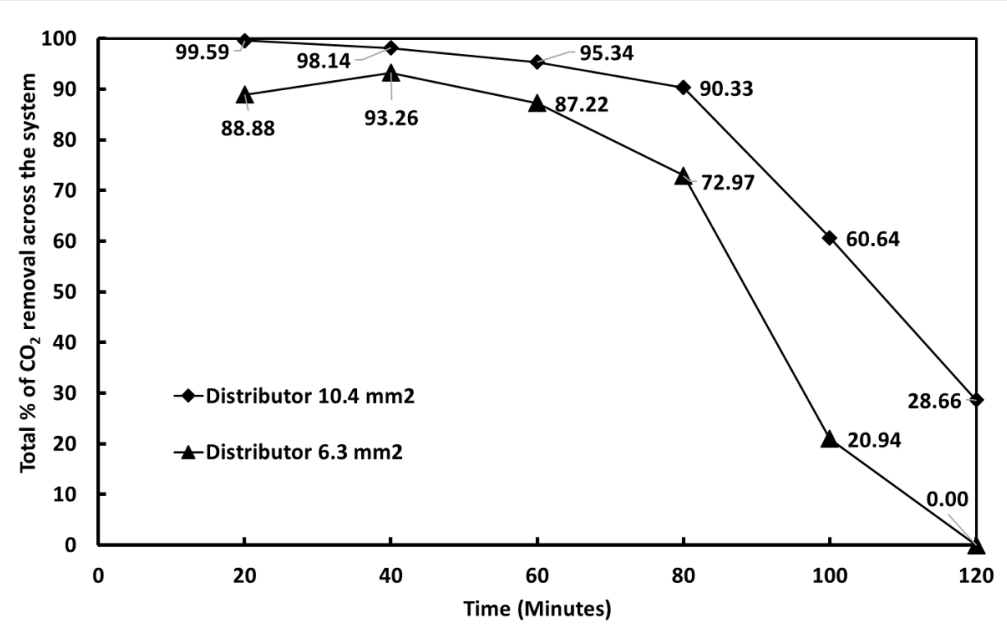

Fig. 5. Total $\mathrm{CO}_{2}$ removal by utilising packing at amine wash towards $\mathrm{CO}_{2}$ removal and the addition of second stage containing 20 wt. $\% \mathrm{NaOH}$. 


\section{Conclusion}

In general, this work shows that a two-stage chemical absorption system using MDEA activated with $\mathrm{PZ}$ and $\mathrm{NaOH}$ has successfully purified the fermentation product of $\mathrm{CO}_{2}$ and $\mathrm{H}_{2}$ mixture up to $99 \mathrm{~mol} \%$ hydrogen purity. Furthermore, the process is feasible to be carried out at ambient pressure and temperature.

The authors acknowledge the support by GUP UTM research grant (Q.J130000.2546.17H90) for funding the research.

\section{References}

1. D. Debabrata, K. Namita, N. Chitralekha Dasgupta. Biohydrogen Production Fundamentals and Technology Advances (2014).

2. F.M Morsy, J. Energy, $\mathrm{CO}_{2}$-free biohydrogen production by mixed dark and photofermentation bacteria from sorghum starch using a modified simple purification and collection system, 87, 594-604, (2015).

3. A. Murugan, A.S. Brown, I.J Hydene, Review of purity analysis methods for performing quality assurance of fuel cell hydrogen, 40(11), 4219-4233, (2015).

4. A Kohl, R. Nielsen, Gas Purification - Alkanolamines for Hydrogen Sulfide and Carbon Dioxide Removal, 40-73, (1997)

5. F. Bauer, C. Hulteberg, T. Persson, D. Tamm, SGC Rapport, Biogas upgrading-Review of commercial technologies, 83, (2013)

6. N. Hidayu A. Rani, N. Muda, $5^{\text {th }}$ International Conference on Environment 2015, Enhancement of Methyldiethanolamine (MDEA absorption capacity for CO2 capture using amine activators, (2015)

7. A.Y. Ibrahim, F.H. Ashour, A.O. Ghallab, M. Ali, JNGSE, Effects of piperazine on carbon dioxide removal from natural gas using aqueous methyl diethanol amine, 21, 894-899, (2014).

8. R.S Alvis, N.A Hatcher, R.H Weiland, J. Rd,. Presented at Nitrogen + Syngas 2012, 20-23 February 2012, Athens, Greece, $\mathrm{CO}_{2}$ Removal from Syngas Using Piperazine - Activated MDEA and Potassium Dimethyl Glycinate, 20-23, (2012). 